J3eA, Journal sur l'enseignement des sciences et technologies de l'information et des systèmes, Volume 2, Hors-Série 2, 2 (2003)

DOI : http://dx.doi.org/10.1051/bib-j3ea:2003602

(C) EDP Sciences, 2003

\title{
Microsystèmes à actionnement électrostatique: conception, modélisation et caractérisation
}

H. Camon, J.-Y. Fourniols, S. Muratet et B. Estibals

Laboratoire d'Analyse et d'Architecture des Systèmes (LAAS-CNRS)

7 avenue du Colonel Roche

F-31077 Toulouse Cedex 04, France

fourniolselaas.fr, camonelaas.fr 


\title{
Microsystèmes à actionnement électrostatique: conception, modélisation et caractérisation
}

\author{
Henri CAMON, Jean-Yves FOURNIOLS, Sylvaine MURATET, Bruno ESTIBALS. \\ Laboratoire d'Analyse et d'Architecture des Systèmes (LAAS-CNRS) \\ 7, avenue du Colonel Roche - 31077 TOULOUSE Cedex 04 \\ E-mail: fourniols@laas.fr, camonelaas.fr
}

\begin{abstract}
Résumé
Depuis une quinzaine d'année les actionneurs électrostatiques sur silicium ont été développés grâce à l'introduction de nouvelles technologies comme le micro-usinage. Certaines réalisations comme les micro-moteurs, sans trouver d'applications immédiates, ont été pionnières dans le domaine. Les micromiroirs, bien que développés plus récemment, ont trouvé un champ d'application plus rapidement (projection, télécommunication). Cet article présente les méthodes et résultats obtenus sur ces deux exemples au sein de notre laboratoire.
\end{abstract}

\section{Introduction}

L'apparition des technologies de micro-usinage de volume et de surface a permis de réaliser dans et à partir des matériaux à base de silicium des structures passives ou actives [1]. Le développement des structures de type capteur a été relativement rapide. Nous citerons pour exemple les capteurs de pression et d'accélération qui ont atteint le stade de la production industrielle. Le développement des actionneurs par ces techniques est plus récent mais il représente un enjeu tout aussi important sur le long terme.

Les actionneurs peuvent faire appel à plusieurs principe: électrique, magnétique, thermique, optique, acoustique et chimique. L'une des plus anciennement développée est l'actionnement électrostatique sur lequel porte cette présentation.

Les déplacements recherchés sont obtenus par l'attraction coulombienne entre charges de signes opposées. La génération de la force $F$ est directement reliée à l'énergie stockée $E$, proportionnelle à la capacité $C$, fortement sensible à la distance entre armatures.

$$
\mathrm{E}=1 / 2 \mathrm{CV}^{2} \text { et } F=-\nabla \mathrm{E}
$$

Pour obtenir des forces exploitables sous des tensions de plusieurs centaines de volts, la distance entre armatures doit être la plus faible possible. La limite est donnée par le champ de claquage (dans l'air, environ $3.10^{4} \mathrm{~V} / \mathrm{cm}$ sur $1 \mathrm{~cm}$ mais $>110^{6} \mathrm{~V} / \mathrm{cm}$ dans la gamme $\left.1-500 \mu \mathrm{m}\right)[2,3]$. On peut remarquer qu'aux dimensions du micron, la densité de puissance mécanique est comparable à celles des systèmes magnétiques rendant de fait la réalisation par des procédés découlant de la microélectronique très attractif.
Cet article s'appuiera sur deux exemples d'actionneur électrostatique (micro-moteur, micro-miroir) pour illustrer des étapes de fabrication, de modélisation et de test.

\section{Micro-moteur électrostatique.}

\subsection{Technologies de fabrication et principe de fonctionnement}

La réalisation d'un micro-moteur électrostatique vers la fin des années 80 à Berkeley [4,5] a été un événement qui illustra spectaculairement les potentialités des technologies de micro-usinage de surface, caractérisé par la fabrication de structures à partir de films minces. Originellement employés pour la fabrication de circuits intégrés, les matériaux utilisés sont des poly-siliciums fortement dopés pour être conducteurs, des oxydes et nitrures de silicium. En utilisant la sélectivité de gravure entre ces matériaux, une libération de couches peut être réalisée, permettant la fabrication de structures tridimensionnelles auto assemblées. La figure 1 illustre les principales étapes de la fabrication. La première couche structurale de poly-silicium sert de plan de masse, la deuxième pour la réalisation du stator et du rotor, la dernière pour le verrou central jouant le rôle d'axe. Nous pouvons d'ailleurs remarquer, qu'aujourd'hui, la majorité des fonderies industrielles ou académiques offrant du Multi Project Wafer, comme CRONOS, propose du micro-usinage de surface à trois couches structurales.

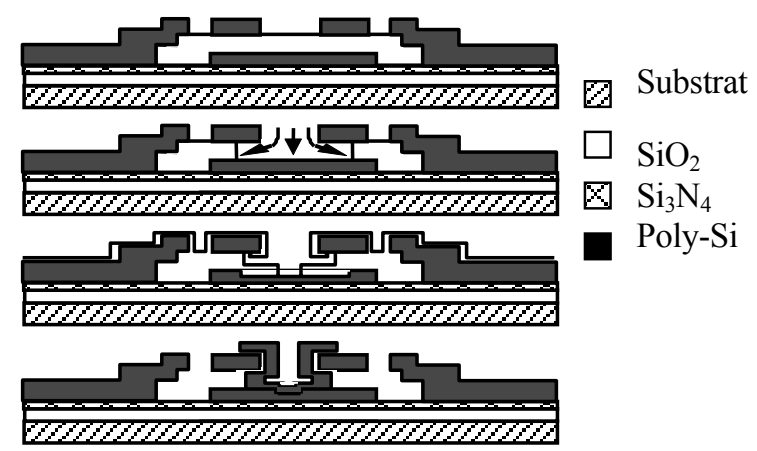

Figure 1. principales étapes de fabrication

Cette structure générique est constituée d'une structure extérieure fixe comportant les électrodes du stator et une 
structure interne formant le rotor, en rotation libre autour d'un axe central assurant une liaison au substrat. Les électrodes du stator sont alimentées individuellement. Le rotor est mis à la masse. La liaison électrique vers une électrode extérieure connectée au plan de masse est assurée par contact soit au niveau l'axe central soit par l'intermédiaire de bossages sous le rotor (fig.2).

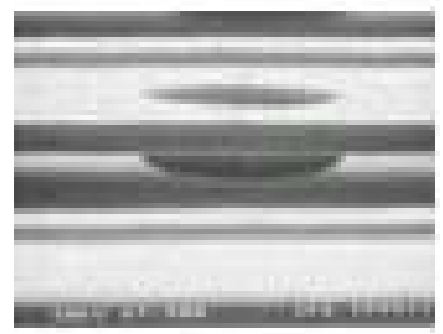

Figure 2 Vue d'un bossage de rotor

Les électrodes du stator sont alimentées suivant trois phases comme indiquées sur la figure 3 pour une structure de type 3/2 (3 électrodes au stator pour 2 électrodes au rotor). La rotation s'opère dans le sens opposé de circulation des phases suivant un fonctionnement de type pas à pas (succession de position fixe obtenue par alignement des pales du rotor avec les électrodes de stator alimentées). La force utile est tangentielle et dépend de la variation de capacité obtenue entre la position intermédiaire et la position alignée.
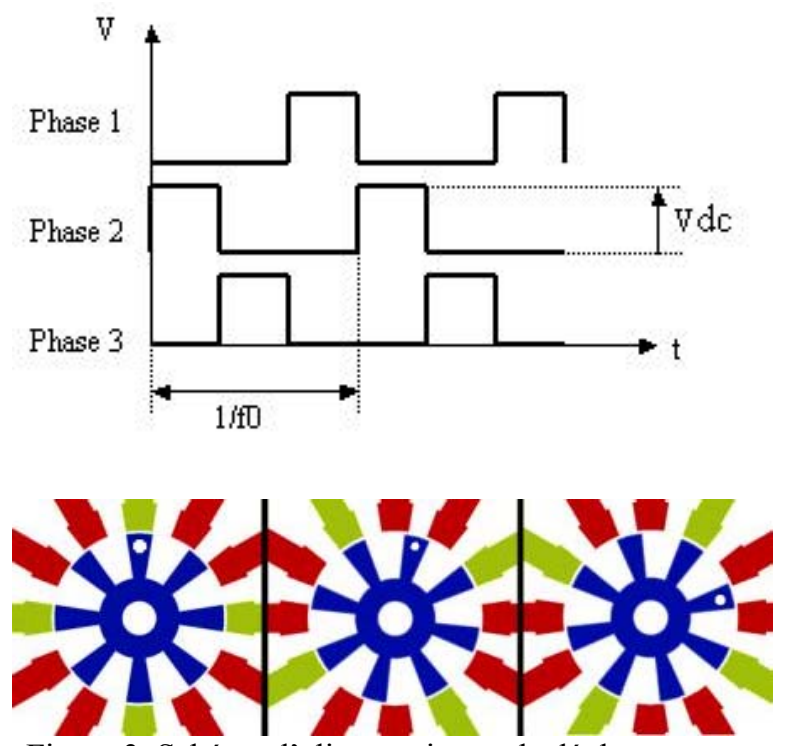

Figure 3. Schéma d'alimentation et de déplacement avec trois phases

Dans les structures décrites précédemment, l'entrefer est nécessairement inférieur au jeu mécanique existant entre le diamètre intérieur du rotor et l'axe central de rotation. C'est le contraire pour des structures dites Wobble ou à rotation désaxée. La composante radiale de la force, bien plus importante que la composante tangentielle, est alors utilisée et le mouvement résulte par le roulement du rotor sur le stator. Le centre du rotor effectue un mouvement décrivant une cycloïde amenant un effet réducteur.
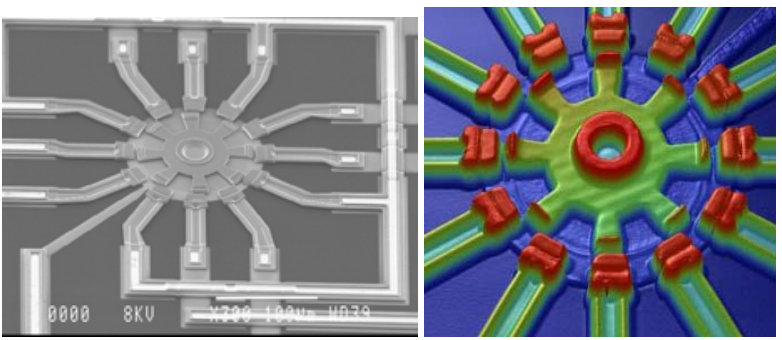

Figure 4: micro-moteur de type 3/2

Ces deux types de structures ont été réalisés en utilisant les potentialités de la fonderie CRONOS [6]. Les figures 4 et 5 illustrent deux des structures réalisées par une image au MEB et une cartographie des altitudes optique.

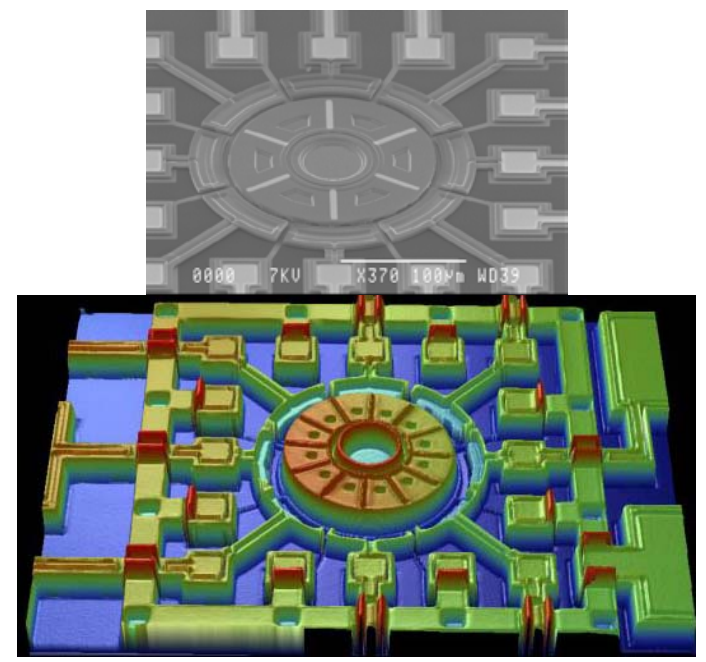

Figure5: micro-moteur de type Wobble

\subsection{Modélisation des micro-moteurs à capacité radiale}

Le rotor est soumis à un ensemble de forces: une force radiale d'attraction du rotor ne participant pas au mouvement, la composante tangentielle motrice et des couples de frottements visqueux dépendant du milieu et secs au niveau des appuis sur l'axe et les bossages.

Les structures présentées sont constituées de 12 électrodes au stator et 8 au rotor, répétant 4 fois une structure $3 / 2$. Le couple moteur est modélisé suivant la formule $[7,8,9]$ :

$$
\Gamma_{p h}(\theta)=\frac{2 \cdot V_{2 \varepsilon 0 \varepsilon r} R_{\text {roh }}}{g} \frac{\partial f_{\text {overlap }}(\theta)}{\partial \theta}
$$

où $V$ est la tension appliquée entre le rotor et le stator, $R_{r o}$ est le rayon externe du rotor, $h$ est l'épaisseur du rotor, $g$ est l'entrefer et $\mathrm{f}_{\text {overlap }}(\theta)$ est la fonction transcrivant la variation de l'angle de recouvrement entre le rotor et le stator. Le comportement dynamique sera déterminé par l'équation suivante:

$$
\ddot{J}=\sum \Gamma_{p h}(\theta)-B \dot{\theta}-\left(C_{1}+C_{2} V_{2}\right) \cdot \operatorname{sign} \dot{\theta}
$$

où $\mathrm{J}$ est l'inertie du rotor, $\mathrm{B}$ le coefficient de frottement visqueux, $\mathrm{C}_{1}$ une constante représentant le frottement sec mécanique et $\mathrm{C}_{2}$ pour ceux induits par des effets de charges au niveau de l'axe.

La modélisation totale est développée en VHDL-AMS dans une architecture quatre blocs comme l'illustre la figure 
6 : le bloc de génération de tension continue Vdc, le bloc de génération des déphasages des trois signaux de commande, le bloc de calcul de couple et le bloc de modélisation dynamique donnant la position $\theta(\mathrm{t})$.

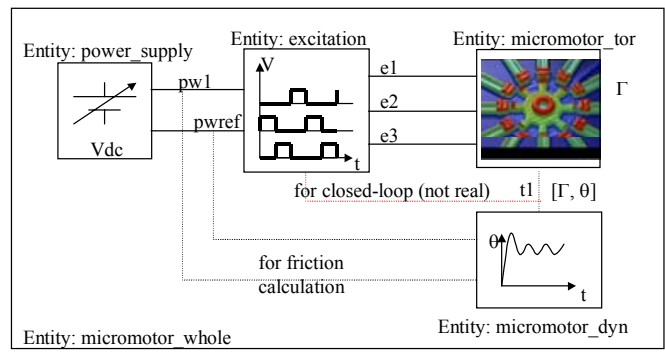

Figure 6 : Diagramme des quatre blocs

La confrontation à l'expérience a été faite avec des mesures de tension de démarrage en fonction de la vitesse de rotation en utilisant des structures réalisées au LAAS [10] et fonctionnant dans une huile silicone isolante. Le protocole de test expérimental (fixation de la fréquence d'excitation, montée progressive de la tension jusqu'à obtention de la rotation) correspond à un fonctionnement en boucle ouverte du schéma précédent.

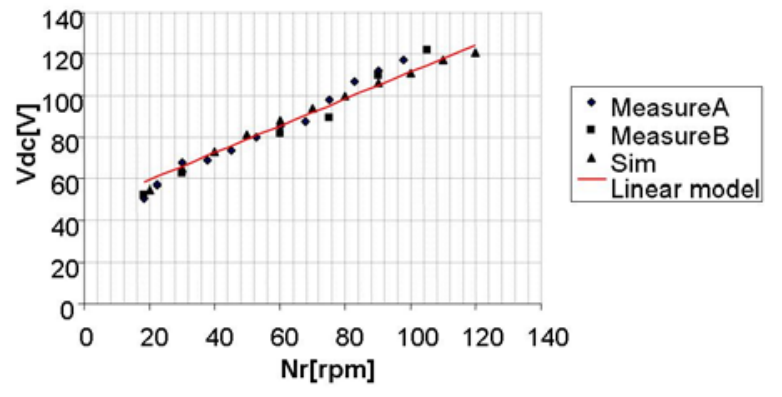

Figure 7. Simulation et expérience : tension de démarrage fonction de la vitesse de rotation en pas à pas

Les valeurs utilisées dans ce cas sont : $\mathrm{B}=2,8 \mathrm{pNm} / \mathrm{s}, \mathrm{C} 1=2$ $\mathrm{pNm}, \mathrm{C} 2=0,0013 \mathrm{pNm} / \mathrm{V}^{2}$. Pour une fréquence d'excitation donnée, quand la tension appliquée est insuffisante, le rotor effectue une oscillation autour d'une position alignée à une électrode du stator. En augmentant la tension, l'amplitude de l'oscillation augmente jusqu'à obtenir la rotation à la tension de démarrage. Ceci correspond au fait que le rotor arrive en position à temps avant le changement de phase. Un surplus de tension accélère le rotor qui atteint la position suivante avant le changement de phase. Il adopte un régime d'oscillation amortie autour de cette nouvelle position d'équilibre.

\section{Micro-miroir électrostatique.}

\subsection{Généralités}

Quelle que soit l'application visée, le système se compose généralement d'une plaque mince réfléchissante, suspendue par des barres de torsion encastrées aux deux extrémités $[11,12]$. Sous cette structure, et placées de part et d'autres de l'axe des barres, sont localisées les électrodes de commande (fig 8.). Les forces électrostatiques engendrées par une tension appliquée entre une électrode et le miroir permettent à celui-ci de pivoter autour de l'axe des barres de torsion. Pour une application de positionnement, une tension sur une électrode entraînera le positionnement du miroir dans sa position tiltée. En excitant alternativement les deux électrodes, le système peut être mis en vibration. En outre, en pilotant le dispositif à sa fréquence propre, on bénéficie d'un facteur d'amplification pour l'angle de balayage égal au facteur de qualité du système $Q$.

Si l'on considère que l'angle de rotation mécanique est $\theta$, l'angle de balayage du faisceau laser est $4 \theta$. En effet, la réflexion sur le miroir incliné à $\theta$ double l'angle de déviation. L'inclinaison du miroir dans l'autre sens multiplie encore par deux cette valeur.

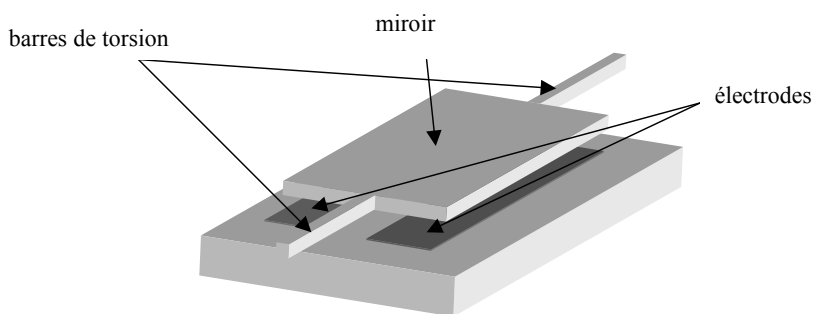

Figure 8. Figure général d'une structure

Un tel dispositif peut être considéré comme un système à un degré de liberté -pendule de torsion. Certains moments s'appliquant sur la partie mobile s'opposent au mouvement:

- le moment d'inertie. Il est proportionnel à l'accélération angulaire et dépend de l'inertie de rotation $I$ de la plaque autour des barres : $\ddot{I \theta}$

- le moment de rappel mécanique. Il dépend de l'angle de rotation: $M m(\theta)=2 k \theta$

- le moment de frottement. On admet qu'il est proportionnel à la vitesse de rotation angulaire et dépend du coefficient de frottement $F: F \dot{\theta}$

Un seul moment ne s'oppose pas au mouvement :

- le moment électrostatique. Il dépend de l'angle de rotation et de la géométrie de l'espace inter-électrode: $\mathrm{M}_{\mathrm{e}}(\theta)$

En écrivant que les moments en présence s'annulent, nous obtenons le modèle suivant :

$$
I \ddot{\theta}+F \dot{\theta}+2 k \theta=\mathrm{M}_{\mathrm{e}}(\theta)
$$

\subsection{Modélisation de la partie électrostatique}

\subsubsection{Cas des faibles angles de rotation}

Pour obtenir des micro-miroirs à faibles angles de déviation, une configuration planaire des électrodes est souvent utilisée. Considérons le schéma de principe de la structure décrit ci-dessous, avec les différentes conventions utilisées:

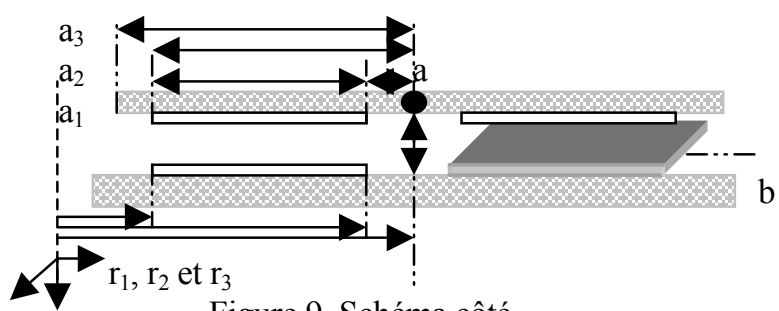

Figure 9. Schéma côté 
La figure précédente montre le principe du micro actionneur, incluant la plaque mise en rotation, les électrodes de commande constituant une capacité variable, ainsi que les diverses notations nécessaires à l'étude du problème.

Dans le but de modéliser le moment électrostatique, considérons une plaque de longueur $L$ dans la direction $z$. Le potentiel du miroir est imposé par une source de tension référencée à la masse ou non. Si $V$ est la tension appliquée au miroir et $\alpha$ représente l'angle maximal entre les deux plans, et en considérant de faibles variations angulaires, le moment électrostatique est donné par :

$$
\vec{M}(\alpha)=\frac{\varepsilon_{0} V^{2} L}{2 \cdot \alpha^{2}} \cdot\left[\frac{d}{d-a_{2} \cdot \alpha}-\frac{d}{d-a_{1} \cdot \alpha}+\ln \left(\frac{d-a_{2} \cdot \alpha}{d-a_{1} \cdot \alpha}\right)\right] \cdot \hat{z}
$$

dans laquelle $d$ est la distance entre les plaques, au niveau de l'axe de rotation, $a_{l}$ est la distance entre l'axe de rotation et le début de l'électrode supérieure et $a_{2}$ la distance jusqu'au fond de l'électrode supérieure. Pour obtenir l'expression de la tension de commande en fonction de l'angle de rotation, il suffit d'égaliser les moments mécaniques et électrostatiques. On obtient alors :

$$
2 K . \alpha=\frac{\varepsilon_{0} \cdot V^{2} L}{2 \alpha_{\max }^{2} \cdot \theta^{2}} \cdot\left[\frac{1}{1-\beta . \theta}-\frac{1}{1-\gamma \cdot \theta}+\ln \left(\frac{1-\beta . \theta}{1-\gamma . \theta}\right)\right]
$$

dans laquelle $\mathrm{K}$ est la raideur de la barre de torsion, dépendant de la nature du matériau utilisée (isotrope ou anisotrope). L'expression de $V$ en fonction de $\alpha$ et $\theta$ est :

$$
V^{2}=\frac{4 K \cdot \alpha^{3}}{\varepsilon_{0} \cdot L} \cdot\left[\frac{1}{1-\beta \cdot \theta}-\frac{1}{1-\gamma \cdot \theta}+\ln \left(\frac{1-\beta \cdot \theta}{1-\gamma \cdot \theta}\right)\right]^{-1}
$$

Les graphiques suivants présentent l'évolution des tensions de commande relatives à un miroir de $700 \mu \mathrm{m}$ par $700 \mu \mathrm{m}$. Ces barres sont ensuite couplées au miroir précédent, afin de tracer leurs caractéristiques statiques. Les figures 10 et 11 montrent les résultats obtenus pour deux angles $\alpha$ proches, respectivement égaux à 0.7 et 0.6 degrés.

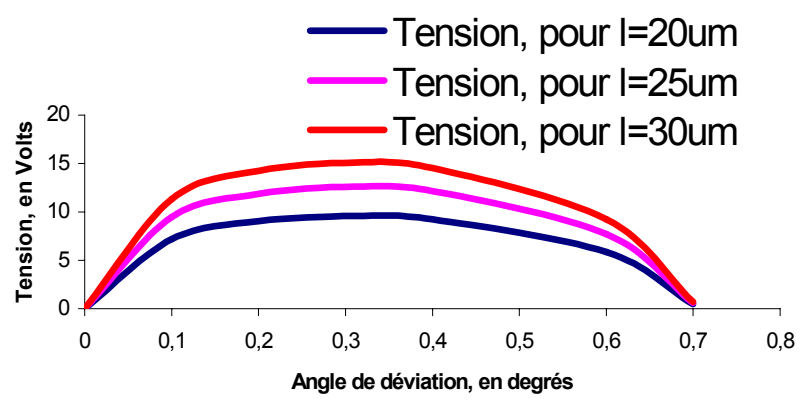

Figure 10. Tension d'actionnement en fonction de l'angle de déviation du miroir. Angle maximal de déviation: $0.7^{\circ}$

On remarque dans un premier temps que la tension d'actionnement augmente avec la raideur du ressort et avec l'angle de déviation maximal. Chaque caractéristique de tension passe alors par un maximum avant de décroître vers zéro.

Comme la masse mobile, en l'occurrence le miroir, est suspendue par deux barres de torsion, cette dernière subit un couple mécanique s'opposant au couple électrostatique.
L'angle de rotation du miroir dérive de l'égalisation des expressions de ces deux moments. Comme on le voit dans la courbe de la figure 11, un cas spécial apparaît et est appelé tension de "pull-in ». Cette valeur de la tension correspond au moment où le couple électrostatique devient supérieur au couple mécanique, causant une attraction du miroir jusqu'au contact avec le premier obstacle sur sa trajectoire.

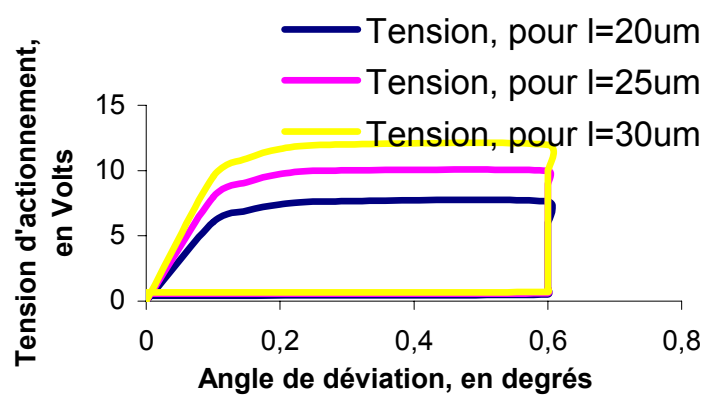

Figure 11. Tension d'actionnement en fonction de l'angle de déviation du miroir (angle maximal de $0,6^{\circ}$ )

La figure 12 illustre des mesures expérimentales sur des structures de miroir de grande taille en commutation, c'est à dire soumis à une impulsion de quelques millisecondes pour des valeurs de tensions très proche du pull-in et au-delà. Le miroir part directement sur l'électrode (inclinée dans ce cas) et reste au contact tant que la tension est maintenue. Une fois la tension remise à la référence, il revient à sa position en oscillation amortie à sa fréquence propre. Le comportement global du miroir correspond à celui d'un système du second ordre.

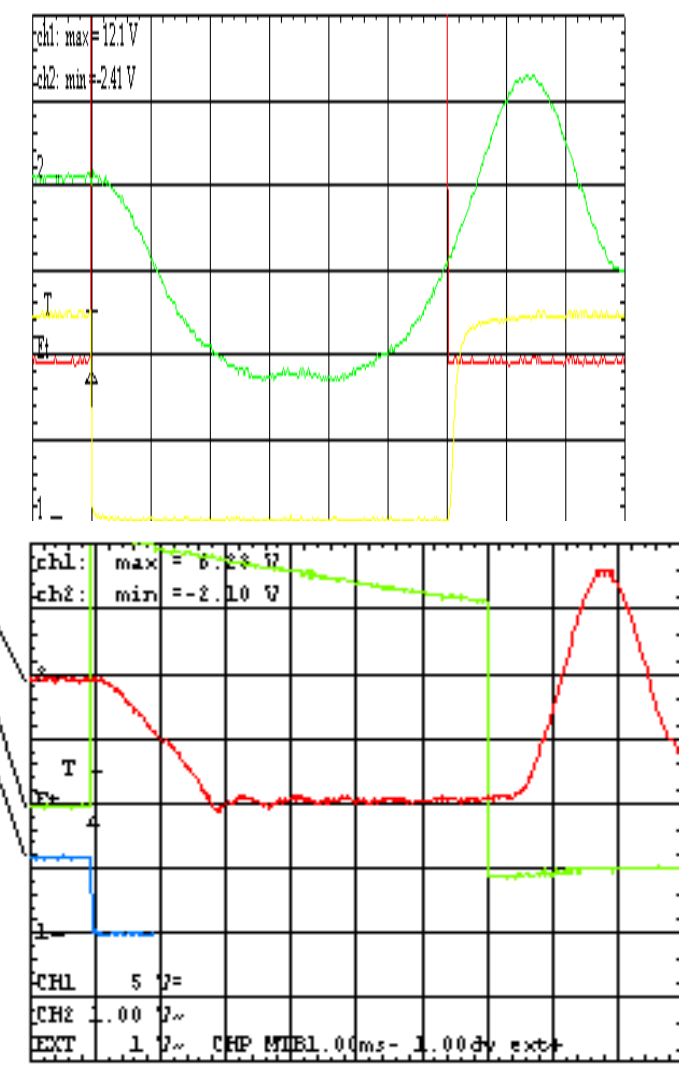

Figure12. Trace du faisceau réfléchi pour $\mathrm{V}$ proche $\mathrm{du}$ pull-in (vert à gauche) et au-delà (rouge à droite), $\theta=10^{\circ}$ 
Le temps de commutation en mode pull-in est variable et dépend beaucoup de l'inertie mise en jeu et des angles. Cela peut aller de quelques dizaines de micro secondes pour des miroirs de petite taille $\left(<1 \mathrm{~mm}^{2}\right)$ à faible angle jusqu'à 1 ou 2 ms pour de grande taille (plusieurs $\mathrm{mm}^{2}$ ) et grand angle. L'utilisation d'une tension nettement supérieure au pull-in permet de diminuer le temps de commutation.

\subsubsection{Cas des forts angles de rotation}

L'inconvénient majeur de l'actionnement électrostatique est la nécessité d'utiliser des fortes tensions pour les grands déplacements du fait de la dépendance de l'interaction électrostatique avec l'inverse du gap au carré. Dans le but de réduire ces tensions, de nombreux travaux ont conduit à la réalisation d'électrodes non-planaires. Les résultats de ces études montrent clairement que l'utilisation de telles électrodes augmente l'interaction électrostatique. L'application d'une tension continue sur une électrode va permettre au miroir de pivoter autour de l'axe des barres. La valeur de l'angle de rotation est calculée en posant:

$$
\frac{d 2 \theta}{d t 2}=\frac{d \theta}{d t}=0
$$

Cet équilibre est en fait atteint lorsque le moment électrostatique compense exactement le moment de rappel des barres de torsion. Les différentes équations conduisent à une relation entre la tension appliquée $V_{0}$ et l'angle de rotation mécanique du miroir $\theta_{0}$ :

$$
V_{0}^{2}=\frac{4 K \theta_{0}\left(\alpha-\theta_{0}\right)^{2}}{\varepsilon_{0} L \ln \left(\frac{d 0}{h \sin \alpha}\right)}
$$

La figure 11 montre le comportement statique d'un micro-miroir réalisé au LAAS-CNRS, dont les caractéristiques géométriques sont: longueur miroir $3 \mathrm{~mm}$, largeur miroir $1 \mathrm{~mm}$, longueur barre $2 \mathrm{~mm}$, largeur barre 10 $\mu \mathrm{m}$. La valeur de l'épaisseur de la structure suspendue $e$, le gap $d_{0}$ sont constants pour tous ces dispositifs $(e=16,5 \mu \mathrm{m}$, $\left.d_{0}=330 \mu \mathrm{m}\right)$. Les valeurs d'angles sont faibles car l'angle des électrodes inclinées est de $35^{\circ}$ dans ce cas de figure. Néanmoins des angles importants peuvent être obtenus mais en commutation comme illustré par les figures 10 et 12. Dans cette dernière figure il s'agit du même miroir que sur la figure 13 mais actionnée sous une tension de $800 \mathrm{~V}$, l'angle de rotation du miroir est alors de $35^{\circ}$.

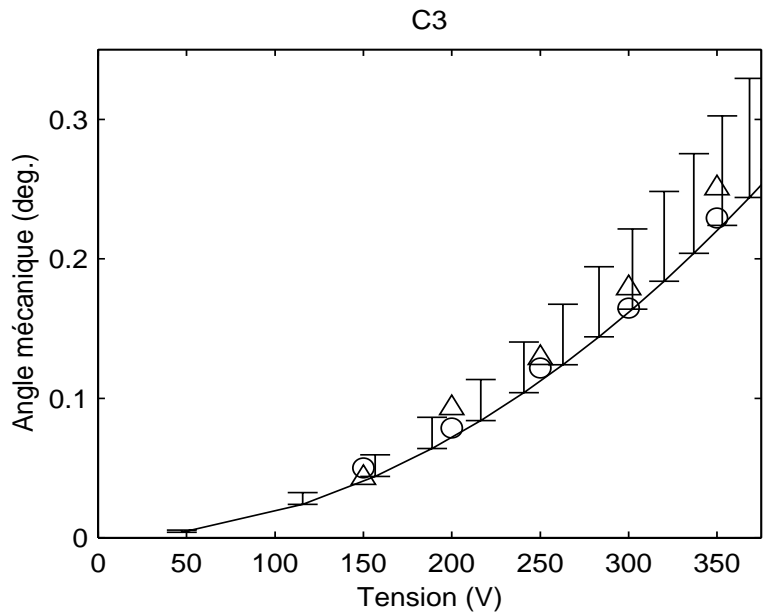

Figure 13. Simulation et expérimentation d'un micro-miroir

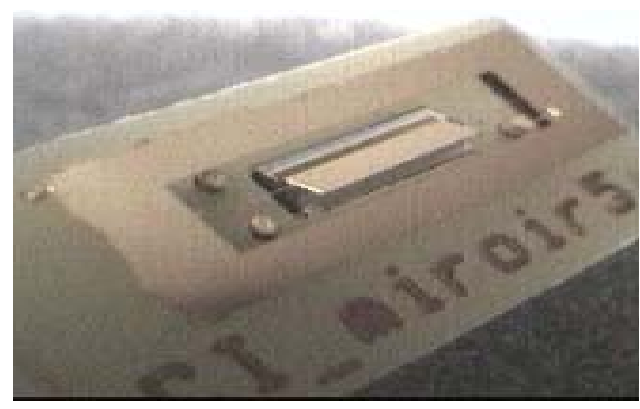

Figure 14. Miroir tilté de $35^{\circ}$

\subsection{Comportement en balayage}

L'application d'une tension alternative au niveau des électrodes permet de mettre en vibration le dispositif. Cette tension de commande intervient dans l'expression de moment électrostatique. Ainsi, en implémentant les différents modules de cette équation dans des logiciels de simulation comme SABER, il est possible d'obtenir les réponses pour différentes sources d'excitation: signal carré, sinusoïdal, triangulaire... Notons tout de même que l'actionnement est non linéaire: en effet, le moment électrostatique dépend de la position du miroir.

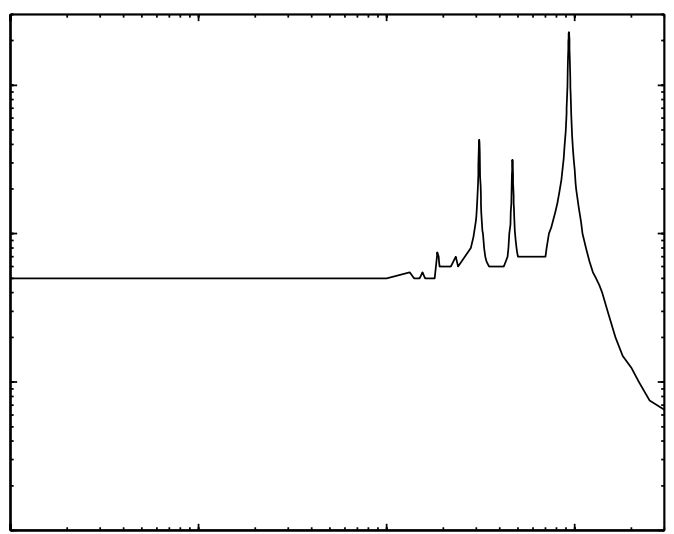

Figure 15. Réponse en fréquence d'une structure

Nous avons représenté sur la figure 15 l'évolution du comportement de torsion d'un dispositif pour une fréquence d'excitation du générateur de tension $V_{\mathrm{e}}$ dans la gamme 1 $\mathrm{Hz}-2,5 \mathrm{kHz}$. Le pic principal correspond à la résonance fondamentale $f_{r}$ de torsion de l'actionneur. Il apparaît également des pics secondaires, pour des fréquences plus basses correspondant à $f_{r} / 2, f_{r} / 3 \ldots$ Nous avons noté qu'en excitant la structure à ces fréquences, celle-ci oscillait à la fréquence $f_{r}$. Nous pouvons donc affirmer que l'origine de ces pics est attribuée à la présence des harmoniques $2 f_{r}, 3 f_{r} \ldots$ dans le spectre du signal de commande.

L'excitation sinusoïdale considérée est constituée d'un signal périodique dont la partie positive est dirigée sur une électrode et la partie négative sur l'autre. Cet argument couplé au fait que le caractère sinusoïdal de la forme n'est pas rigoureusement respecté, explique l'apparition des harmoniques.

$\mathrm{Au}$ final, nous pouvons dire que, quand la fréquence excitatrice est très faible, l'amplitude de balayage du 
résonateur est très peu différente de celle du comportement statique. Quand la fréquence excitatrice est égale à la fréquence propre du résonateur, l'amplitude est maximale. Pour des fréquences d'excitation très supérieures à la fréquence propre du système, l'amplitude de vibration forcée redevient très faible.

De ce fait, notre micro dispositif peut être assimilé à un filtre mécanique ne réagissant qu'à des fréquences égales à sa fréquence propre de torsion. Ainsi, la réponse du dispositif à un signal donné dépend de l'amplitude de la raie de son spectre fréquentiel correspondant à la fréquence de résonance mécanique.

Nous noterons que l'angle de balayage peut être important (jusqu'à $100^{\circ}$ optique sous $240 \mathrm{~V}$ à $950 \mathrm{~Hz}$ ) pour la structure de la figure 14 par exemple, et cela à la pression atmosphérique. En effet, les pertes sont très majoritairement dues à l'amortissement visqueux. Les facteurs de qualité sont typiquement pour ces dimensions de miroir de 70 à 80 à la pression ambiante et augmente légèrement jusqu'à 10 mbar. L'expérience montre que si l'on diminue la pression du milieu ambiant, des facteurs de qualités de plusieurs milliers sont rapidement atteints en dessous du mbar. L'évolution du facteur de qualité est alors régie par les impacts moléculaires. A ces faibles pressions, des angles importants sont alors obtenus avec une tension réduite d'autant.

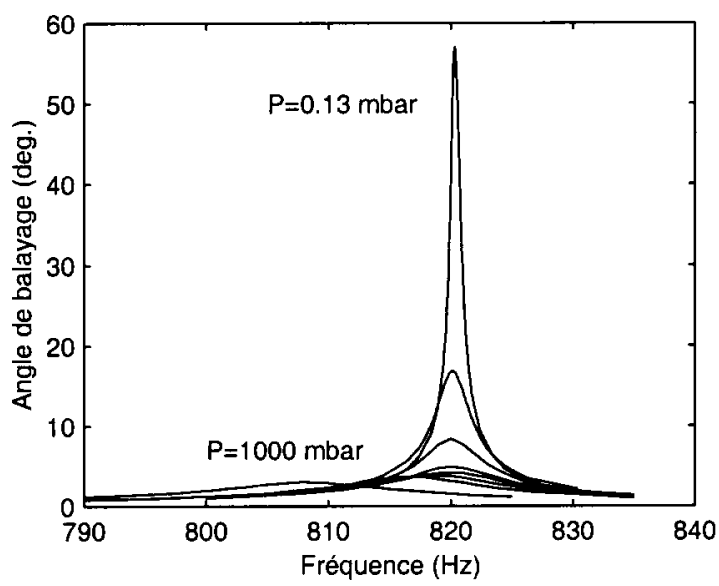

Figure 16. exemple de réponse dynamique en fonction de la pression ambiante pour un même tension d'excitation

\section{Conclusion}

Le développement des actionneurs électrostatiques à partir des technologies silicium a été historiquement plus lent que celui des capteurs et reste un sujet d'étude tant au niveau de la fabrication et de la modélisation. Certaines réalisations sont plus proches de l'application comme les micro-miroirs aussi bien pour des systèmes de projection (Matrice Texas Instruments) que pour les Optical Cross Connect en télécommunication. Il est a noté également l'apparition récente de fonderie pour micro-systèmes en micro-usinage de surface à trois couches structurales poly-silicium (CRONOS) ou à une couche structurale utilisant du SOI (Tronic's Microsystems SA) permettant des réalisations pour des laboratoires ou sociétés non spécialisés en technologie. Ces éléments indiquent clairement que l'approche des microsystèmes, dont les actionneurs électrostatiques ne sont qu'une part, tend à se développer identiquement à la microélectronique dans le passé.
Remerciements: Les auteurs remercient les organismes ayant supporter les études sur les micro-moteurs (CNRS, CNES) et les micro-miroirs (CNRS, Tronics Microsystems SA) ainsi que l'ensemble de l'équipe TEAM du LAAS pour leur support opérationnel en fabrication.

\section{Références}

[1] L. Fan, Y. Tai, R. Müller, «Ic-processed electrostatic micromotors», IEEE IEDM, pp. 666-669, 1988.

[2] W.S.N. Trimmer, K.J. Gabriel, «Design considerations for practical electrostatic micromotors», Sensors and Actuators, vol. 11, pp. 189-206, 1987

[3] T.W. Dakin \& al., «Breakdown of gases in uniform fields», Electra (32), pp. 61-82, 1974.

[4].L. Fan, Y. Tai, R. Müller, «Ic-processed electrostatic micromotors», IEEE IEDM, pp. 666-669, 1988.

[5] S.D. Senturia, «Simulation and design of Microsystems: a 10year perspective», Sensors \& Actuators, vol. A 67, pp. 1-7, 1998.

[6] D.A. Koester \& al., «Multi-user MEMS process (MUMP's) design handbook», Cronos Integrated Miceosystems, available at http://www.memsrus.com/mumps.pdf

[7] E. Sarraute, «Etude et modélisation de microactionneurs integers sur silicium», Thèse Inst. Nat. PolyTechnique, Toulouse, Octobre 1993.

[8] K. Deng, G.P. Ramanathan, M. Mehregany, «Micromotors dynamics in lubricating fluids», J. of Micromechanics and Microengineering, vol. 4, pp. 266-269, 1994.

[9] J.Y. Founiols, A. Endemano, H. Camon, M. Desmulliez, A. Marchese, S. Muratet, F. Bony, «VHDL-AMS modelling, simulation and test of oplanar electrostatic micromotors: application to MEMS virtual prototyping», to be published.

[10] V. Conédéra, N. Fabre, H. Camon, B. Rousset, «Firt steps toward the fabrication of electrostatic micromotors using SOG», Sensors \& Actuators, vol. A 46-47, pp. 82-84, 1998

[11] F. Larnaudie, «Micromiroir rotatif à actionnement électrostatique en silicium: de la conception à la réalisation», Thèse Univ. P. Sabatier, Toulouse, 2000.

[12] B. Estibals, «Conception, réalisation et caractérisation de micro-miroirs optiques à déflexion localisée appliqués aux télécommunications optiques», Thèse de l'INPT soutenue le 19 Décembre 2002

[13] H. Camon, F. Larnaudie, "Fabrication, simulation and experiment of a rotating electrostatic silicon mirror with large angular deflection", Proceedings of the IEEE MEMS'2000, pp. 645650,2000 . 